\title{
SESAM fabrication errors and its influence on ultrafast laser cavity design
}

\author{
Sha WANG ${ }^{1}$, Guo-ying FENG ${ }^{\dagger 11}$, Shou-huan ZHOU ${ }^{\dagger 1,2}$ \\ ( ${ }^{1}$ Institute of Laser \& Micro/Nano Engineering, College of Electronics and Information Engineering, \\ Sichuan University, Chengdu 610064, China) \\ ( ${ }^{2}$ North China Research Institute of Electro-Optics, Beijing 100015, China) \\ †E-mail: guoingfeng@scu.edu.cn; zhoush@scu.edu.cn
}

Received Feb. 15, 2015; Revision accepted Mar. 9, 2015; Crosschecked Mar. 25, 2015

\begin{abstract}
During mode-locked ultrafast laser experiments, we find that semiconductor saturable absorber mirrors (SESAMs) from the same manufacturing process may, from batch to batch, show different working ranges: pure Q-switching, Q-switched mode-locking, and continuous wave $(\mathrm{CW})$ mode-locking. This is because, in high-volume wafer-scale fabrication, there is typically an estimated 1\% error for high-quality molecular beam epitaxy (MBE) growth, which introduces a variation in the parameters of an individual SESAM. In this paper, we will analyze how that $1 \%$ error in layer thickness influences the behaviour of SESAMs in three different structures: resonant SESAM, anti-resonant SESAM, and enhanced SESAM. Furthermore, the characteristics of the SESAM will affect the mode-locking dynamic behavior of ultrafast solid state lasers. In the worst case, a SESAM with a fabrication error may prevent the laser cavity from mode-locking. Proper laser cavity design can help to reduce the impact of SESAM fabrication errors on laser performance and maintain the laser in the $\mathrm{CW}$ mode-locking range.
\end{abstract}

Key words: Semiconductor saturable absorber mirror (SESAM), Ultrafast laser, Fabrication error, Design criteria doi: $10.1631 /$ jzus.A1500030

\section{Introduction}

Mode-locked femtosecond lasers have been developed rapidly since their introduction 30 years ago (Mollenauer and Stolen, 1984). To get self-start and maintain the stability of the mode-locked ultrafast laser, a saturable absorber is normally required inside the laser cavity (Kartner and Keller, 1995; Kartner et al., 1996; Keller et al., 1996). Originally, dyes were the saturable absorbers for mode-locking systems. However, they are toxic and degrade in long term running (Kubecek et al., 2001). A solid state saturable absorber, such as Cr:YAG, V:YAG, normally has very long recovery time (from a few to hundreds of nanoseconds), and thus cannot be used for femto-

\footnotetext{
Corresponding author

(ii) ORCID: Sha WANG, http://orcid.org/0000-0002-7038-1828; Guo-ying FENG, http://orcid.org/0000-0002-4533-1423

(c) Zhejiang University and Springer-Verlag Berlin Heidelberg 2015
}

second lasers (Malyarevich et al., 1998; Sulc et al., 2004). The semiconductor saturable absorber mirror (SESAM), a relative newly invented saturable absorber, is a very reliable and widely used source for both solid state ultrafast lasers and fiber ultrafast lasers. A semiconductor working as a saturable absorber has several advantages (Keller et al., 1996; Saraceno, 2012). First, the semiconductor material has bi-temporal response time, the fast intraband thermalization helps to self-start mode-locking, while the slow interband recombination helps to stabilize it. Second, by changing the composition of semiconductor, it is possible to change the band gap, i.e., change the absorption wavelength, which makes the SESAM suitable for different desired working wavelengths. Third, and one of the most important features of SESAM, is that the semiconductor saturable absorber is grown in a Bragg mirror structure. Different Bragg mirror structure designs can 
influence the characteristics of the absorber, such as saturation fluence, modulation depth, and the group delay dispersion (GDD) of the SESAM. By manipulating the parameters of these devices, it is possible to control the working of the laser at different laser operation regions, i.e., pure passive Q-switching, pure continuous wave $(\mathrm{CW})$ mode-locking, or Q-switched mode-locking (Keller et al., 1992; 1993; Keller, 1994). When a SESAM is used for CW mode-locking, situations like Q-switched mode-locking and pure passive Q-switching must be suppressed. However, during manufacturing of SESAMs, any manufacturing process will introduce systematic and random errors into the optical thicknesses of layers (Tikhonravov et al., 2006). The thickness error mainly comes from two aspects: inadequacy in the monitoring method and optical constant errors (Sullivan and Dobrowolski, 1992). Thus, the errors in SESAM characteristics could not be avoided, i.e., errors in saturation fluence, modulation depth, and damage threshold cannot be avoided. That is also why when we build ultrafast lasers even with the same fabrication process SESAMs, the laser performance varies from one batch to another. Sometimes the lasers cannot even get mode-locking but instead show pure Q-switching. Although SESAM designs and manufacture errors are widely known, how fabrication errors affect SESAM's optical behavior and how to design a laser cavity which can handle more fabrication errors have, to our knowledge, never been discussed in detail before.

In this paper, we will first use the transfer matrix method to analyze how fabrication thickness errors influence a SESAM's enhancement factor and GDD. We will find out how these changes in enhancement factor influence the SESAM's modulation depth and saturation fluence. Finally, we will suggest how to design a laser cavity to tolerate fabrication errors in SESAMs.

\section{Influence of fabrication errors on SESAM's enhancement factor and GDD}

Fabrication errors, including inadequacy in monitoring methods and optical constant errors, will result in errors in SESAM layer thickness. It is reported that a $1 \%$ error is a typical value for high quality MBE growth (Rudin et al., 2010), i.e., the typical value for a thickness error is $1 \%$. In this section, we will compare the influence of fabrication errors on three different SESAM structures: antiresonant SESAM, resonant SESAM, and enhanced SESAM.

\subsection{Transfer matrix method}

Before we start to analyze the influence of fabrication thickness errors on the behavior of SESAMs, we will quickly review the transfer matrix method which is used to calculate field intensity distribution, reflectivity, and phase spectrum in a Bragg mirror. Fig. 1 shows the forward and backward propagating waves in the first several layers of a Bragg mirror. This model can be expanded to any number of layers. The field distribution comes from interference between the individual reflections from the dielectric boundary. $k_{\mathrm{i}}=2 \pi n / \lambda$ is the propagation constant, which depends on the wavelength and on the refractive index of the material.

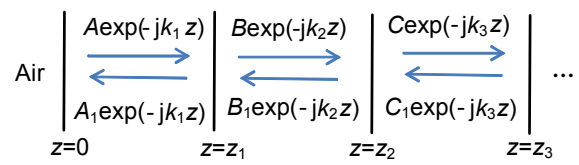

Fig. 1 Propagating waves in a Bragg structure mirror

It is already known that the field amplitudes and their derivatives must be equal at each boundary. Here we assume the amplitude of the input electric field is normalized to 1 , the reflection of the electric field is $r$, and its transmission is $t$.

At the boundary between the air and the first high index layer $z=0$,

$$
\begin{aligned}
& {\left[\begin{array}{cc}
1 & 1 \\
-\mathrm{j} k_{\text {Air }} & \mathrm{j} k_{\text {Air }}
\end{array}\right]\left[\begin{array}{l}
1 \\
r
\end{array}\right]=\left[\begin{array}{cc}
1 & 1 \\
-\mathrm{j} k_{1} & \mathrm{j} k_{1}
\end{array}\right]\left[\begin{array}{l}
A \\
A_{1}
\end{array}\right],} \\
& {\left[\begin{array}{c}
A \\
A_{1}
\end{array}\right]=M_{1}(0)^{-1} M_{\text {Air }}(0)\left[\begin{array}{l}
1 \\
r
\end{array}\right] .}
\end{aligned}
$$

At the first internal boundary,

$$
\begin{aligned}
& {\left[\begin{array}{cc}
\exp \left(-\mathrm{j} k_{1} z_{1}\right) & \exp \left(\mathrm{j} k_{1} z_{1}\right) \\
-\mathrm{j} k_{1} \exp \left(-\mathrm{j} k_{1} z_{1}\right) & \mathrm{j} k_{1} \exp \left(\mathrm{j} k_{1} z_{1}\right)
\end{array}\right]\left[\begin{array}{l}
A \\
A_{1}
\end{array}\right]} \\
& \quad=\left[\begin{array}{cc}
\exp \left(-\mathrm{j} k_{2} z_{1}\right) & \exp \left(\mathrm{j} k_{2} z_{1}\right) \\
-\mathrm{j} k_{2} \exp \left(-\mathrm{j} k_{2} z_{1}\right) & \mathrm{j} k_{2} \exp \left(\mathrm{j} k_{2} z_{1}\right)
\end{array}\right]\left[\begin{array}{l}
B \\
B_{1}
\end{array}\right],
\end{aligned}
$$




$$
\left[\begin{array}{c}
B \\
B_{1}
\end{array}\right]=M_{2}\left(z_{1}\right)^{-1} M_{1}\left(z_{1}\right)\left[\begin{array}{l}
A \\
A_{1}
\end{array}\right]
$$

At the second internal boundary,

$$
\begin{aligned}
& {\left[\begin{array}{cc}
\exp \left(-\mathrm{j} k_{2} z_{2}\right) & \exp \left(\mathrm{j} k_{2} z_{2}\right) \\
-\mathrm{j} k_{2} \exp \left(-\mathrm{j} k_{2} z_{2}\right) & \mathrm{j} k_{2} \exp \left(\mathrm{j} k_{2} z_{2}\right)
\end{array}\right]\left[\begin{array}{l}
B \\
B_{1}
\end{array}\right]} \\
& =\left[\begin{array}{cc}
\exp \left(-\mathrm{j} k_{3} z_{1}\right) & \exp \left(\mathrm{j} k_{3} z_{2}\right) \\
-\mathrm{j} k_{3} \exp \left(-\mathrm{j} k_{3} z_{2}\right) & \mathrm{j} k_{3} \exp \left(\mathrm{j} k_{3} z_{2}\right)
\end{array}\right]\left[\begin{array}{l}
C \\
C_{1}
\end{array}\right], \\
& {\left[\begin{array}{c}
C \\
C_{1}
\end{array}\right]=M_{3}\left(z_{2}\right)^{-1} M_{2}\left(z_{2}\right)\left[\begin{array}{c}
B \\
B_{1}
\end{array}\right] .}
\end{aligned}
$$

In the similar way, we can get the total system matrix of the coating

$$
\begin{aligned}
{\left[\begin{array}{l}
t \\
0
\end{array}\right]=} & M_{N}\left(z_{n-1}\right)^{-1} M_{N-1}\left(z_{n-1}\right) \cdots \\
& M_{2}\left(z_{1}\right)^{-1} M_{1}\left(z_{1}\right) M_{1}(0)^{-1} M_{\text {Air }}(0)\left[\begin{array}{l}
1 \\
r
\end{array}\right]
\end{aligned}
$$

From Eq. (4), we have

$$
\begin{aligned}
& {\left[\begin{array}{l}
t \\
0
\end{array}\right]=\left[\begin{array}{ll}
M_{11} & M_{12} \\
M_{21} & M_{22}
\end{array}\right]\left[\begin{array}{l}
1 \\
r
\end{array}\right],} \\
& r=-\frac{M_{21}}{M_{22}} \\
& t=M_{11}+M_{12} r .
\end{aligned}
$$

From the transfer matrix method we can calculate the reflectivity spectrum $R=|r|^{2}$ and the field distribution in the stack

$$
E(n)=E_{1} \exp \left(-\mathrm{j} k_{n} z\right)+E_{2} \exp \left(\mathrm{j} k_{n} z\right)
$$

where $E_{1}$ and $E_{2}$ are the amplitude coefficients of the electric field for that layer. From Eqs. (1)-(8), we can obtain the electric field distribution along the stack, the wavelength dependent reflectivity and phase spectrum. The second derivation of the phase is called dispersion.

When consider thickness errors in SESAM Bragg mirror fabrication theoretically, we introduce a random error in $z$ position for each layer and rewrite Eq. (2) as

$$
\left[\begin{array}{l}
B \\
B_{1}
\end{array}\right]=M_{2}\left[z_{1}+e\left(z_{1}\right)\right]^{-1} M_{1}\left[z_{1}+e\left(z_{1}\right)\right]\left[\begin{array}{l}
A \\
A_{1}
\end{array}\right],
$$

where $e\left(z_{1}\right)$ represents the layer thickness error. It should be the same for all other layers.

Now we will discuss how thickness errors influence the characteristics of a SESAM. For each SESAM design, we apply less than $1 \%$ growth error of different materials for 100 random structures.

\subsection{Anti-resonant SESAM}

Anti-resonant SESAM is usually defined as the structure in which the standing wave inside has a node at the front surface of the structure, and typically exhibits features such as small GDD and little change of enhancement factor throughout a wide range of operation wavelengths, and good growth tolerance which makes the fabrication insensitive to growth errors. It has very low field enhancement and suffers relatively high saturation fluence.

Fig. 2 shows the layer structure and field intensity of a $1040 \mathrm{~nm}$ anti-resonant design SESAM. Here $1040 \mathrm{~nm}$ means that the SESAM is designed for a center wavelength at $1040 \mathrm{~nm}$. We assume 28 pairs of alternating quarter-wave layers of AlAs as low-index material and GaAs as high-index material in order to create more than $99.9 \%$ reflectivity. Incoming light with intensity normalized to 1 creates a standing wave pattern in this structure; the peak of the field intensity of the resulting standing wave pattern is then calculated as 4 outside the device. Ideally, without any thickness growth error, a saturable absorber is located at the maximum field intensity position in the first GaAs layer, and the enhancement factor $\xi$ is defined as the intensity at the saturable absorber (Spuhler et al., 2005). However, if we consider a thickness growth error, the field intensity distribution will be changed, and then the parameters such as enhancement factor and phase, i.e., GDD of the SESAM, will be affected. Fig. 3a shows the electric field enhancement in the absorber as a function of the wavelength, and Fig. $3 \mathrm{~b}$ shows the calculated wavelength dependence of the GDD. For SESAM in an antiresonant design, the enhancement factor and GDD are nearly flat over the center wavelength range.

From Fig. 3, we find that at the designed wavelength, the thickness error does not influence the enhancement factor and GDD of the SESAM very much. For example, at its center wavelength $1040 \mathrm{~nm}$, if there is no growth error, the enhancement factor is 


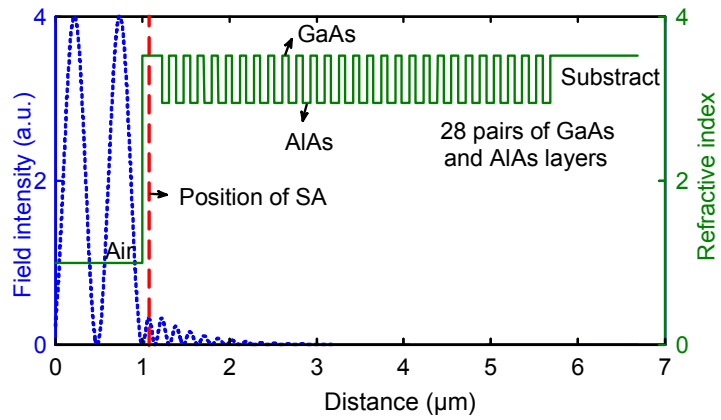

Fig. 2 Refractive index structure (solid) and calculated standing wave intensity pattern for $\lambda=1040 \mathrm{~nm}$ (dot) of a $1040 \mathrm{~nm}$ anti-resonant design SESAM. Note that the node of standing wave is located at the surface of the device which is located at $1.04 \mu \mathrm{m}$ in the figure
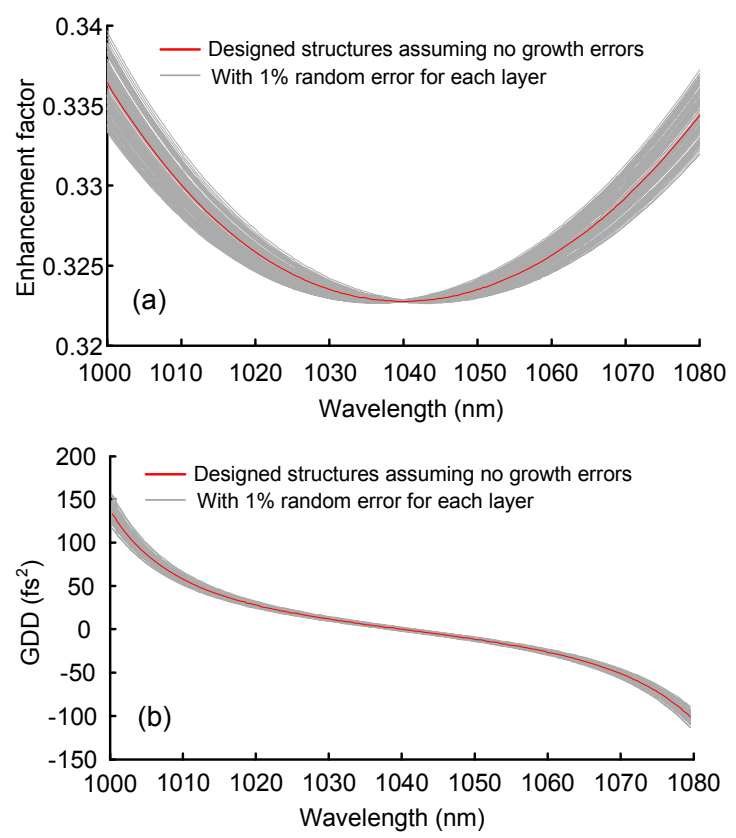

Fig. 3 Fabrication errors induced: (a) enhancement factor change and (b) GDD change of an anti-resonant SESAM

calculated as 0.3228 and the GDD value is calculated as 0 . When a $1 \%$ thickness error is applied, the enhancement factor error is calculated at $\pm 0.031 \%$, and the GDD error at $\pm 2.5 \mathrm{fs}^{2}$. Obviously those errors are very small. However, if this SESAM is set working at other wavelengths, the error increases. For example, at $1030 \mathrm{~nm}$, the enhancement factor is 0.3235 and the enhancement error increases to $\pm 0.22 \%$; the GDD increases to 12.1 , and the GDD error is $\pm 3 \mathrm{fs}^{2}$. The further the operation wavelength is from the designed center wavelength, the larger the error becomes.

\subsection{Resonant SESAM}

The surface of the SESAM structure is positioned at the anti-node of the optical field, the characteristics of resonant structures are typically opposite to those of anti-resonant cases. They exhibit violent fluctuations in both GDD and enhancement factor as a function of operation wavelength, high sensitivity to growth errors, high scattering loss, and low damage threshold. Usually there is a high field enhancement factor inside the device, which results in low saturation fluence.

Fig. 4 shows the layer structure and field intensity of a $1040 \mathrm{~nm}$ resonant design SESAM. For a resonant SESAM, the enhancement factor is as large as 4. Relative to the anti-resonant SESAM described above, the field enhancement factor is more than 12 times larger. This implies that the saturation fluence should be 12 times lower and the modulation depth 12 times higher. We also calculated the wavelength dependent enhancement factor and GDD (Figs. 5a and $5 b)$.

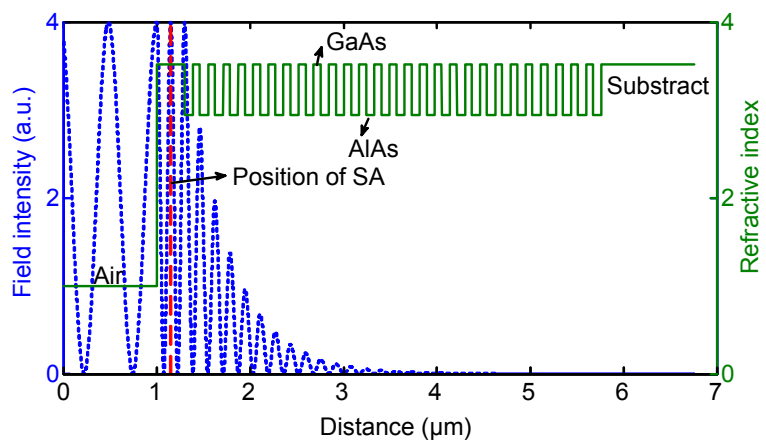

Fig. 4 Refractive index structure (solid) and calculated standing wave intensity pattern for $\lambda=1040 \mathrm{~nm}$ (dot) of a $1040 \mathrm{~nm}$ resonant design SESAM. Note that the antinode of the standing wave is located at the surface of the device

As shown in Fig. 5, we find that at the designed wavelength, the thickness error does not influence SESAM's enhancement factor very much, but the GDD value will be affected by the growth error. At center wavelength of $1040 \mathrm{~nm}$, the enhancement factor is calculated as 4, and the GDD value is calculated as $-45.64 \mathrm{fs}^{2}$ if there is no growth error. When a $1 \%$ thickness error is applied, the enhancement factor error is calculated as $-0.875 \%$, which is slightly larger than that for an anti-resonant SESAM. We 
should mention here that for a resonant SESAM, the enhancement factor can never be larger than 4 , so the enhancement error can only be negative. Moreover, the GDD error increases to $\pm 381 \mathrm{fs}^{2}$, which cannot be ignored when designing an ultrafast laser cavity. If this SESAM is set working at $1030 \mathrm{~nm}$, the enhancement factor decreases to 3.693 , and the enhancement factor error increases to $\pm 5.74 \%$. From Fig. 5a, we know that the further away from center wavelength, the smaller the enhancement factor and the larger the enhancement factor error are. However, we surprisingly find that the GDD error at $1030 \mathrm{~nm}$ decreases to almost $\pm 125 \mathrm{fs}^{2}$ with GDD of $-915.1 \mathrm{fs}^{2}$. This is because of the resonant behavior of the GDD curve; the smallest GDD error will appear at two GDD peaks near $1028 \mathrm{~nm}$ and $1052 \mathrm{~nm}$.
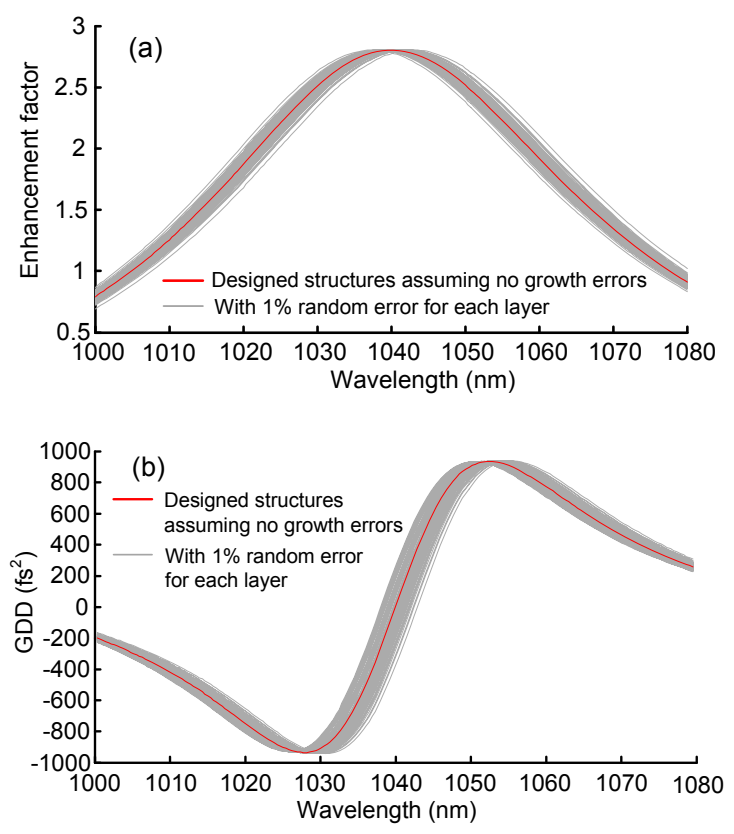

Fig. 5 Fabrication errors induced: (a) enhancement factor change and (b) GDD change of a resonant SESAM

\subsection{Enhanced SESAM}

The enhanced SESAM (Spuhler et al., 2005) is an intermediate solution between an anti-resonant SESAM and a resonant SESAM. An enhanced SESAM has a single low-index (i.e., lower than the low-index (distributed Bragg reflector (DBR) material) dielectric quarter-wave layer deposited as a cap layer. In our analysis here, we deposit a quarter-wave layer of $\mathrm{SiN}_{4}$ at the surface (Fig. 6).

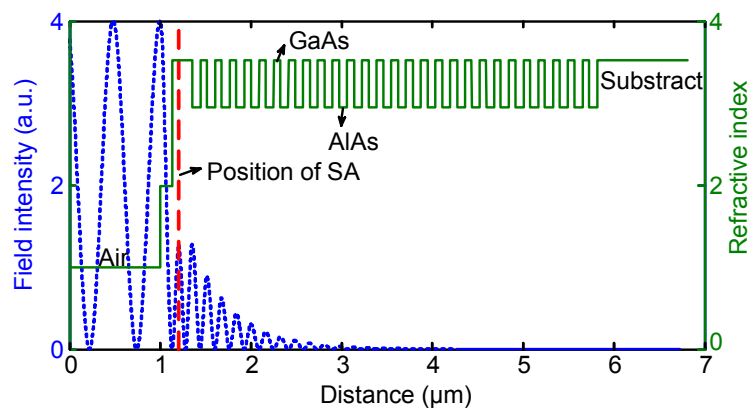

Fig. 6 Refractive index structure (solid) and calculated standing wave intensity pattern for $\lambda=1040 \mathrm{~nm}$ (dot) of a $1040 \mathrm{~nm}$ enhanced SESAM

The enhanced SESAM has a relatively high enhancement factor of 1.013, about one quarter of the resonant SESAM, but still more than three times enhanced compared to the anti-resonant SESAM device. As shown in Fig. 7, the wavelength dependence of enhancement factor is strongly reduced, and the GDD of this structure is now nearly wavelength independent and close to zero at the center wavelength.
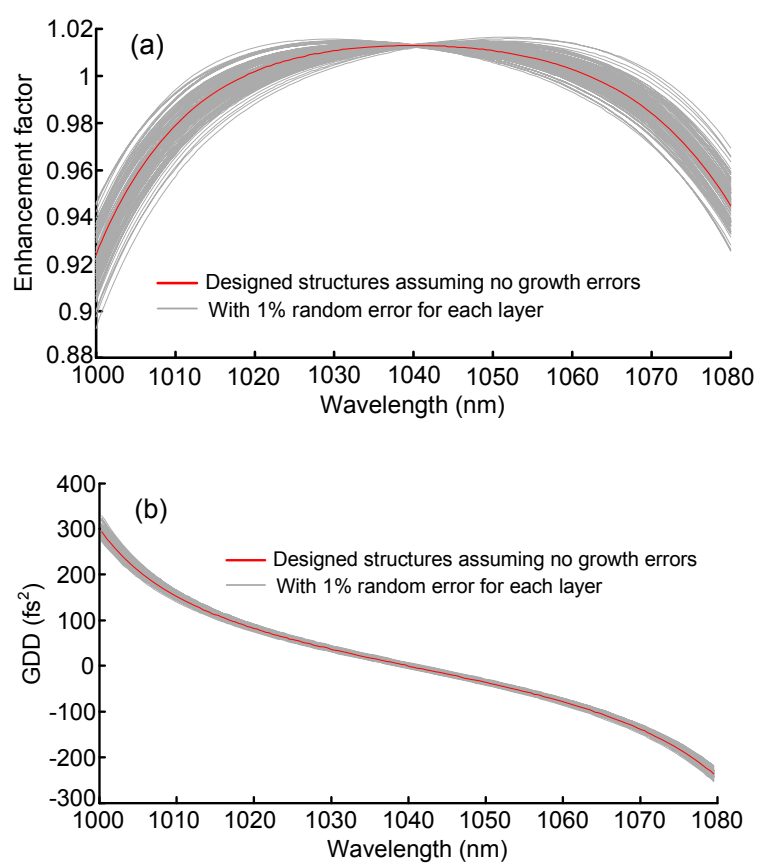

Fig. 7 Fabrication errors induced: (a) enhancement factor change and (b) GDD change of an enhanced SESA

Enhanced SESAM is almost the same as antiresonant SESAM, in that the thickness error does not influence the enhancement factor $( \pm 0.1 \%)$ and GDD $\left( \pm 7 \mathrm{fs}^{2}\right)$ very much when the center wavelength is 
$1040 \mathrm{~nm}$. When working at $1030 \mathrm{~nm}$, the enhancement factor decreases to 1.011 , and the enhancement factor error increases to $\pm 0.69 \%$; the GDD value increases to $36.3 \mathrm{fs}^{2}$, and the GDD error increases to $\pm 8 \mathrm{fs}^{2}$.

\section{Influence of fabrication errors on SESAM's saturation fluence and modulation depth}

We have shown that a fabrication error will introduce errors in enhancement factor and GDD value. In this section, we will discuss how the enhancement factor error affects the optical characteristics of SESAMs. At the designed center wavelength, the enhancement factor error is relatively small for all three kinds of SESAM structure. Working further away from the center wavelength, the enhancement factor error becomes larger. As we know, the enhancement factor determines the saturation fluence and the modulation depth of a SESAM. The product of modulation depth and saturation fluence equals a constant value $F_{\mathrm{t}}$, which is the transparency fluence of the absorber (Saraceno, 2012). $F_{\mathrm{t}}$ is related to the characteristics of the quantum well (QW) or quantum dot (QD) absorber. Once the density of QW or QD and the thickness of absorber are fixed, $F_{\mathrm{t}}$ is a constant number. If we consider the saturable layer only

$$
\Delta R_{\mathrm{SA}} \times F_{\text {sat, SAlayer }}=F_{\mathrm{t}},
$$

where $\Delta R_{\mathrm{SA}}$ is the modulation depth and $F_{\text {sat,SAlayer }}$ is the saturation fluence of the saturable absorber layer. For the SESAM, we have

$$
F_{\text {sat, } \mathrm{A}}=F_{\text {sat,SAlayer }} / \xi,
$$

then

$$
\Delta R=\Delta R_{\mathrm{SA}} \times \xi .
$$

From Eqs. (11) and (12), we know that an enhancement factor error will result in a saturation fluence error $F_{\text {sat,A }}$ and a modulation depth $\Delta R$ error in a SESAM. According to the error transfer function, we have

$$
e\left(F_{\text {sat }, \mathrm{A}}\right)=\frac{e(\xi)}{\xi},
$$

$$
e(\Delta R)=e(\xi)
$$

where $e(\Delta R), e\left(F_{\mathrm{sat}, \mathrm{A}}\right)$, and $e(\xi)$ are errors of modulation depth, saturation fluence, and enhancement factor of SESAM, respectively. Table 1 gives the calculated saturation fluence error and modulation depth error for different SESAM structures according to the previous calculated enhancement factor error and error transfer functions Eqs. (13) and (14).

From Table 1, we know that the saturation fluence and modulation depth of a SESAM will be influenced by manufacturing errors. The saturation fluence error and modulation depth error at the designed center wavelength are small. The further away from the designed center wavelength, the larger the fluence error and the modulation depth error. The saturation fluence error at off-center wavelength is almost the same for an anti-resonant SESAM and an enhanced SESAM, but for a resonant SESAM it is more than twice as large. The modulation depth error at the off-center wavelengths is the smallest for an anti-resonant SESAM and the largest for a resonant SESAM.

Table 1 Summary of the enhancement factor errors and GDD errors of different SESAM structures

\begin{tabular}{rccccc}
\hline \multirow{2}{*}{$\begin{array}{c}\text { SESAM } \\
\text { structure }\end{array}$} & \multicolumn{2}{c}{ Enhancement } & GDD & $\begin{array}{c}\text { GDD error } \\
\left(\mathrm{fs}^{2}\right)\end{array}$ \\
\cline { 2 - 5 } & Factor & Error $(\%)$ & $\left(\mathrm{fs}^{2}\right)$ & \\
\hline \multicolumn{1}{c}{ Anti- } & $1040 \mathrm{~nm}$ & 0.3228 & \pm 0.031 & 0.0 & \pm 2.5 \\
resonant & $1030 \mathrm{~nm}$ & 0.3235 & \pm 0.22 & 12.1 & \pm 3 \\
Resonant $1040 \mathrm{~nm}$ & 4.000 & -0.875 & -45.64 & \pm 381 \\
$1030 \mathrm{~nm}$ & 3.693 & \pm 5.74 & -915.1 & \pm 125 \\
Enhanced $1040 \mathrm{~nm}$ & 1.013 & \pm 0.10 & 0.0 & \pm 7 \\
$1030 \mathrm{~nm}$ & 1.011 & \pm 0.69 & 36.3 & \pm 8 \\
\hline
\end{tabular}

\section{Laser cavity design to accommodate er- rors in SESAM fabrication}

The modulation depth and saturation fluence of a SESAM will affect the dynamic working ranges of an ultrafast laser (Kartner et al., 1995; Honninger et al., 1999). The errors of modulation depth and saturation fluence will change the laser working statues from pure Q-switching, Q-switched mode-locking to $\mathrm{CW}$ mode-locking.

(1) When the Q-switching driving force 
$F_{\mathrm{Q}}=\frac{2 q_{0} \tau_{\mathrm{A}} F_{\text {sat, }} A_{\text {eff }, \mathrm{L}}}{T_{\mathrm{R}} F_{\text {sat }, \mathrm{A}} A_{\mathrm{eff}, \mathrm{A}}}$ is larger than 1 , the laser will only run in the pure Q-switch range. Here, $q_{0}$ is the unsaturated but saturable loss per round trip; $\tau_{\mathrm{A}}$ is the absorber recovery time; $F_{\text {sat, } \mathrm{L}}$ is the saturation fluence of the gain material; $A_{\mathrm{eff}, \mathrm{L}}$ is the area of laser beam at the gain material; $T_{\mathrm{R}}$ is the round trip time; $F_{\text {sat, } \mathrm{A}}$ is the saturation fluence of the saturable absorber; and $A_{\text {eff,A }}$ is the area of the laser beam at the saturable absorber. Large errors in saturation fluence $F_{\text {sat, } \mathrm{A}}$ will change the value of Q-switching driving force, and may force the laser into pure Q-switching range.

(2) When the Q-switched mode locked driving force $F_{\mathrm{QM}}=F_{\text {sat } \mathrm{L}} A_{\text {sat } \mathrm{L}} F_{\text {sat }, \mathrm{A}} A_{\text {sat }, \mathrm{A}} \Delta R / E_{\mathrm{p}}{ }^{2}$ is larger than 1, the laser will run in a $\mathrm{Q}$-switched mode-locking range. Here $E_{\mathrm{p}}$ is the pulse energy. As discussed in (1), the errors in saturation fluence and modulation depth will change the value of the Q-switched mode-locked driving force as well, and may drive the laser to fall into a Q-switched mode-locking range. These two situations mentioned above are what we want to avoid in an ultrafast laser.

In this section, we will discuss how to design an ultrafast laser cavity to tolerate fabrication error. Fig. 8 is a typical ultrafast laser cavity structure. Here for simplification, we do not consider the Brewster angle of the laser crystal. $M_{1}, M_{2}$, and $M_{3}$ are curved mirrors with curvatures $R_{1}, R_{2}$, and $R_{3}$, respectively; the laser crystal has a length $l$; the distances $d_{1}$ and $d_{2}$ between each two optical components are shown in Fig. 8 .

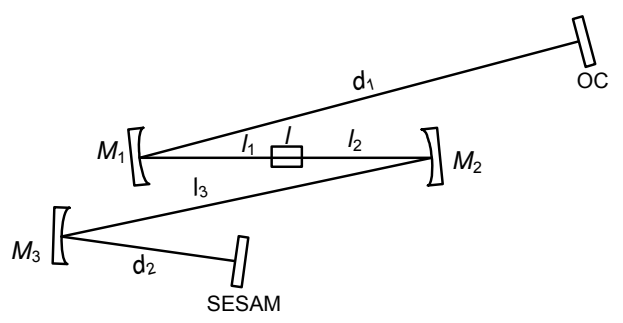

Fig. 8 Setup of an ultrafast laser

The first suggestion we make here is to avoid using a resonant SESAM, because it has the largest modulation depth error, saturation fluence error, and GDD error. If such a SESAM is used, not only do modulation depth and saturation fluence need to be considered, but also a GDD compensation system will be needed inside the cavity. So in reality, we would like to avoid using a resonant SESAM.
The second criterion we propose is to have the laser running at the SESAM's designed wavelength, which will help the laser against fabrication errors. However, sometimes for a specific application, we have to run the laser off its designed center wavelength. In that case, we can design the laser cavity to help tolerate a SESAM fabrication error.

Here we will take a $1030 \mathrm{~nm} \mathrm{Yb:KGW} \mathrm{laser} \mathrm{as}$ an example. The emission cross section of $\mathrm{Yb}: \mathrm{KGW}$ is $2.5 \times 10^{-20} \mathrm{~cm}^{2}$ at $1030 \mathrm{~nm}$, and the saturation fluence of $\mathrm{Yb}: \mathrm{KGW}$ is $F_{\text {sat, } \mathrm{L}}=h v /\left(2 \sigma_{\mathrm{L}}\right)=3.86 \times 10^{4} \mathrm{~J} / \mathrm{m}^{2}$. Normally a slow SESAM will be used for mode-locked Yb-doped lasers. Here we will use Batop SESAM SAM-1040-1-10ps as an example, $q_{0}=1 \%, \tau_{\mathrm{A}}=10 \mathrm{ps}, F_{\mathrm{sat}, \mathrm{A}}=70 \mu \mathrm{J} / \mathrm{cm}^{2}$, and $R=0.6 \%$. Substituting all these values into the Q-switching driving force, we can obtain $F_{\mathrm{Q}}=\frac{1.6543 A_{\mathrm{eff}, \mathrm{L}}}{L_{\mathrm{cav}} A_{\mathrm{eff}, \mathrm{A}}}$, where $L_{\mathrm{cav}}$ is the cavity length of the laser. Considering a fabrication error may make $F_{\mathrm{Q}}$ larger than that with no fabrication error. For an anti-resonant SESAM and an enhanced SESAM, $F_{\mathrm{Q}}$ turns to $F_{\mathrm{Q}}=\frac{1.6655 A_{\text {eff }, \mathrm{L}}}{L_{\mathrm{cav}} A_{\text {eff } \mathrm{A}}}$. Normally $M_{1}, M_{2}$, and $M_{3}$ have the same curvatures, and $A_{\text {eff,L }}$ and $A_{\text {eff, } \mathrm{A}}$ are designed the same, so if we want SESAMs working properly to get mode-locking even with some fabrication error, the smallest laser cavity length should be at least $1.6655 \mathrm{~m}$ in order to make $F_{\mathrm{Q}}$ less than 1 , which corresponds to a repetition rate of $90 \mathrm{MHz}$. If we want to run the laser at higher repetition rate, i.e., shorter cavity length, in this case $A_{\text {eff,L }}$ should be designed to be smaller than $A_{\text {eff,A }}$. For example, if the laser is running at $100 \mathrm{MHz}, A_{\text {eff,L }}$ should be smaller than $0.9 A_{\text {eff, } \mathrm{A}}$, i.e., $w_{\text {eff, } \mathrm{L}}$ should be smaller than $0.95 w_{\text {eff,A }}$.

Now we discuss the Q-switched mode-locked driving force $F_{\mathrm{QM}}=F_{\text {sat }, \mathrm{L}} A_{\mathrm{sat}, \mathrm{L}} F_{\mathrm{sat}, \mathrm{A}} A_{\mathrm{sat}, \mathrm{A}} \Delta R / E_{\mathrm{p}}{ }^{2}$, introducing a fabrication error into an anti-resonant SESAM will make $F_{\mathrm{QM}}{ }^{\prime}=1.009 F_{\mathrm{QM}}$ and into an enhanced SESAM $F_{\mathrm{QM}}{ }^{\prime}=1.0137 F_{\mathrm{QM}}$. From Table 1, it is obvious that an anti-resonant SESAM is the best structure to mitigate a fabrication error in this case. If we want to operate the laser at $\mathrm{CW}$ mode-locking even with fabrication error and off its designed center wavelength, the only solution is to make the laser beam area in the gain material smaller or increase the pulse energy by pumping harder. 
In conclusion, for a given repetition rate ultrafast laser, we should choose the curvature $R_{3}$ of the mirror $M_{3}$ to be larger than the curvatures $R_{1}=R_{2}$ of the curved mirrors $M_{1}$ and $M_{2}$. For example, to design a $100 \mathrm{MHz} \mathrm{CW}$ mode-locking laser with $\mathrm{Yb}: \mathrm{KGW}$ crystal and Batop SESAM SAM-1040-1-10ps SESAM, we could choose curvatures of $M_{1}$ and $M_{2}$ at $75 \mathrm{~mm}, M_{3}$ at $100 \mathrm{~mm}, d_{1}=1071 \mathrm{~mm}, l_{1}=37 \mathrm{~mm}$, $l_{2}=37 \mathrm{~mm}, l_{3}=300 \mathrm{~mm}, l=3 \mathrm{~mm}$, and $d_{2}=50 \mathrm{~mm}$. In that case the beam diameter in the laser crystal is calculated at $24 \mu \mathrm{m}$, and at SESAM is calculated as $30 \mu \mathrm{m}$. For such a design, we need not worry about Q-switching induced by a fabrication error. If, in addition, we put all these numbers into a Q-switched mode-locked driving force, we can obtain the result that if the pulse energy $E_{\mathrm{p}}$ is higher than $4.05 \mathrm{~nJ}$, that is $40.5 \mathrm{~mW}$ power for a $100 \mathrm{MHz}$ laser system, we can avoid Q-switched mode-locking. If we consider $10 \%$ optical-optical conversion efficiency, that is a pump power of $405 \mathrm{~mW}$. For such a laser cavity design, as long as we have more than $405 \mathrm{~mW}$ pump power, the fabrication error will not affect the performance of the laser.

Fortunately, because the GDD errors caused by anti-resonant SESAM and enhanced SESAM are small, we do not need to consider a GDD compensation system inside the cavity.

\section{Discussion}

During experiments, we find that it is a normal problem for SESAM that there is much difference between different manufactured batches. But people seldom discuss how SESAM fabrication error affects its optical parameters and performance. Rudin et al. (2010) analyzed how the fabrication error in layer thickness changes the GDD and enhancement factor in a mode locked integrated external-cavity surface emitting laser (MIXSEL). They showed that the antiresonant design has a substantially higher growth error tolerance than the resonant one. However, they did not investigate the possibility of tolerating errors by laser cavity design, which may because that MIXSEL is a chip laser.

However, for a solid state ultrafast laser using SESAM as a mode-locking device, we can, by laser cavity design during the manufacturing process, al- ways tolerate the actual thickness error in maintaining the mode-locking function. We use a transfer matrix method to analyze how the layer thickness errors in SESAM affect the optical characteristics. We also find out that resonant SESAM is not a good option because it needs additional GDD control components and other extra work to compensate if we are seeking an "error free" mode-locking laser. Compared with resonant SESAM, anti-resonant SESAM or enhanced SESAM itself can reduce the fabrication error to a certain degree; the GDD error in these two structures is in generally very small and can be ignored. But the errors in modulation depth and saturation influence may still prevent the ultrafast laser from modelocking. In Section 3, we propose that by proper laser cavity design, mode-locking function can be maintained and thickness error can be tolerated from the manufacturing process. It could be a guiding method later for people designing an ultrafast laser cavity to tolerate more SESAM fabrication errors.

\section{Conclusions}

In this paper, we discuss how the fabrication thickness error affects the characteristics of three SESAM structures: anti-resonant SESAM, resonant SESAM, and enhanced SESAM. From theoretical calculations, we find that at the designed center wavelength, a fabrication error does not influence SESAM's modulation depth and saturation fluence that much for all three structures. GDD value is affected a little more in resonant SESAM than in the other two. However, if the SESAM is working at an off-center wavelength, the modulation depth error, saturation fluence error, and GDD error will increase. When designing an ultrafast laser cavity which can tolerate a fabrication error, we should first avoid resonant SESAM, then select the right cavity length (or repetition rate), and make appropriate the curvatures of $M_{1}, M_{2}, M_{3}$ mirrors (beam sizes on SESAM and gain crystal), and the pumping level to operate the laser running in the $\mathrm{CW}$ mode-locking range.

\section{References}

Honninger, C., Paschotta, R., Morier-Genoud, F., et al., 1999. $Q$-switching stability limits of continuous-wave passive mode-locking. Journal of the Optical Society of America $B, \mathbf{1 6}(1): 46-56$. [doi:10.1364/JOSAB.16.000046] 
Kartner, F.X., Keller, U., 1995. Stabilization of soliton like pulses with a slow saturable absorber. Optics Letters, 20(1):16-18. [doi:10.1364/OL.20.000016]

Kartner, F.X., Brovelli, L.R., Kopf, D., et al., 1995. Control of solid state laser dynamics by semiconductor devices. Optical Engineering, 34(7):2024-2036. [doi:10.1117/12. 204794]

Kartner, F.X., Jung, I., Keller, U., 1996. Soliton mode-locking with saturable absorbers. IEEE Journal of Selected Topics in Quantum Electronics, 2(3):540-556. [doi:10.1109/ 2944.571754]

Keller, U., 1994. Ultrafast all-solid-state laser technology. Applied Physics B, 58(5):347-363. [doi:10.1007/ BF01081874]

Keller, U., Miller, D.A.B., Boyd, G.D., et al., 1992. Solid-state low-loss intracavity saturable absorber for Nd:YLF lasers an antiresonant semiconductor Fabry-Perot saturable absorber. Optics Letters, 17(7):505-507. [doi:10.1364/ OL.17.000505]

Keller, U., Chiu, T.H., Ferguson, J.F., 1993. Self-starting femtosecond mode-locked $\mathrm{Nd}$ : glass laser using intracavity saturable absorbers. Optics Letters, 18(13): 1077-1097. [doi:10.1364/OL.18.001077]

Keller, U., Weingarten, K.J., Franz, X., et al., 1996. Semiconductor saturable absorber mirrors (SESAM's) for femtosecond to nanosecond pulse generation in solidstate lasers. IEEE Journal of Selected Topics in Quantum Electronics, 2(3):435-453. [doi:10.1109/2944.571743]

Kubecek, V., Dombrovsky, A., Biegert, J., et al., 2001. Mode-locking of flash lamp pumped Nd:YAP laser using solid state saturable absorbers. Proc. SPIE 4267, Solid State Lasers X, San Jose, CA, p.128-133. [doi:10.1117/ 12.424605]

Malyarevich, A.M., Denisov, I.A., Yumashev, K.V., et al., 1998. V:YAG-a new passive Q-switch for diode-pumped solid-state lasers. Applied Physics B: Lasers and Optics, 67(5):555-558. [doi:10.1007/s003400050544]

Mollenauer, L.F., Stolen, R.H., 1984. The soliton laser. Optics Letters, 9(1):13-15. [doi:10.1364/OL.9.000013]

Rudin, B., Wittwer, V.J., Maas, D.J.H.C., et al., 2010. High-power MIXSEL: an integrated ultrafast semiconductor laser with $6.4 \mathrm{~W}$ average power. Optics Express, 18(26):27582-27588. [doi:10.1364/OE.18.027582]

Saraceno, C.J., 2012. Cutting-edge High-power Ultrafast Oscillators: Pushing the Limits of SESAM Modelocked Thin-disk Lasers. PhD Thesis, Eidgenössische Technische Hochschule ETH Zürich.
Spuhler, G.J., Weingarten, K.J., Grange, R., et al., 2005. Semiconductor saturable absorber mirror structures with low saturation fluence. Applied Physics B, 81(1):27-32. [doi:10.1007/s00340-005-1879-1]

Sulc, J., Jelinkova, H., Nemec, M., et al., 2004. V:YAG saturable absorber for flash lamp and diode pumped solid state lasers. Proc. SPIE 5460, Solid State Lasers and Amplifiers, Strasbourg, France, p.292-302. [doi:10.1117/ 12.544822]

Sullivan, B.T, Dobrowolski, J.A., 1992. Deposition errorcompensation for optical multilayer coatings. I. Theoretical description. Applied Optics, 31(19):3821-3835. [doi:10.1364/AO.31.003821]

Tikhonravov, A.V., Trubetskov, M.K., Amotchkina, T.V., et al., 2006. Investigation of the effect of accumulation of thickness errors in optical coating production by broadband optical monitoring. Applied Optics, 45(27):70267034. [doi:10.1364/AO.45.007026]

\section{中文概要}

题 目: 可饱和吸收镜制造误差及其对飞秒激光器腔型设 计的影响

目 的: 从可饱和吸收镜 (SESAM) 制造误差所引起其光 学特性的改变出发, 提出采用飞秒激光器腔型设 计的方法, 避免 SESAM 误差对激光输出性能造 成的影响。

创新点: 从激光器腔型设计方案出发, 采用不同曲率镜, 调整激光腔中激光晶体及可饱和吸收镜上的光 斑半径大小比值, 解决 SESAM 制造误差对激光 器输出的影响。

方 法: 采用传递矩阵法计算 SESAM 半导体膜层厚度对 其光学性能的影响。对于不同的 SESAM 设计, 影响大小不同（表 1)。

结 论: 通过改变腔内曲率镜 $M_{1}, M_{2}$ 和 $M_{3}$ 的曲率值大小 以及腔的长度值, 可以减弱 SESAM 制造误差对 飞秒激光器输出的影响, 实现对所有 SESAM 的 连续锁模。

关键词: 可饱和吸收镜; 飞秒激光器; 制造误差; 腔型 设计 